\title{
Ulinastatin Attenuates LPS-induced Human Endothelial Cells Oxidative Damage through Suppressing JNK/c-Jun Signaling Pathway
}

\author{
Chunping $\mathrm{Li}^{* 1}$, Dandan Ma*1, Man Chen ${ }^{1}$, Linlin Zhang ${ }^{2}$, Lin Zhang ${ }^{1}$, Jicheng Zhang ${ }^{1}$, Xin Qu ${ }^{1}$, Chunting Wang ${ }^{1 凶}$ \\ 1.Department of Critical-care Medicine, Shandong Provincial Hospital affiliated to Shandong University, Jinan250021, China
}

2.Department of Critical-care Medicine, Affiliated Hospital of Weifang Medical University,Weifang261000,China

*These authors contributed to the work equally and should be regarded as co-first authors.

${ }^{\square}$ Corresponding author: Chunting Wang

phone: $+86-13370517000$

Email: wcteicu@126.com

Fax: +86-0531-68773083

Address all correspondence and requests for reprints to: Department of Critical-care Medicine, Shandong Provincial Hospital,

9677 Jing 10 Rd, Jinan, 250101 Shandong China

Abbreviations: LPS. Lipopolysaccharide; UTI. Ulinastatin; HUVECs. human umbilical vein endothelial cells; ROS. reactive oxygen species ; SOD. superoxide dismutase ; MDA. Malondialdehyde; JNK. c-Jun N-terminal kinases; MAPKs. mitogen-activated protein kinases; ERKs. extracellular signal-regulated kinases 


\section{Abstract}

Lipopolysaccharide (LPS)-induced oxidative stress is a main feature observed in the sepsis by increasing endothelial oxidative damage. Many studies have demonstrated that Ulinastatin (UTI) can inhibit pro-inflammatory proteases, decrease inflammatory cytokine levels and suppress oxidative stress. However, the potential molecular mechanism underlying UTI which exerts its antioxidant effect is not well understood. In this study, we aimed to investigate the effects of UTI on the LPS-induced oxidative stress and the underlying mechanisms using human umbilical vein endothelial cells (HUVECs). After oxidative stress induced By LPS in HUVECs, the cell viability and reactive oxygen species (ROS) in cytoplasm were measured. In addition, superoxide dismutase (SOD) and malondialdehyde (MDA) were examined. We found that LPS resulted in a profound elevation of ROS production and MDA levels. The decrease in $\mathrm{Cu} / \mathrm{Zn}-\mathrm{SOD}$ protein and increased in Mn-SOD protein were observed in a time- and dose-dependent manner. These responses were suppressed by an addition of UTI. The increase in c-Jun N-terminal kinases (JNK) phosphorylation by LPS in HUVECs was markedly blocked by UTI or JNK inhibitor SP600125. Our results suggest that UTI exerts its anti-oxidant effects by decreasing overproduction of ROS induced by LPS via suppressing JNK/c-Jun phosphorylation. Therefore UTI may play a protective role in vascular endothelial injury induced by oxidative stress such as sepsis. This study may provide insight into a possible molecular mechanism by which Ulinastatin inhibits LPS-induced oxidative stress.

Keywords: JNK/c-Jun signaling pathways, Oxidative stress, Ulinastatin, Lipopolysaccharide, HUVECs, ROS 


\section{Introduction}

Severe sepsis is a leading cause of intensive care unit and its incidence is rapidly increasing annually. The pathogenesis of sepsis is complex and oxidative stress is one of the key features of sepsis [1]. Oxidative stress is usually defined as the imbalance of oxidant production and antioxidant defenses, which results in cellular dysfunction and tissue damage [2].LPS, a major component of the Gram-negative bacteria cell wall, can trigger and accelerate oxidative stress [3], which has been widely used to mimic features of oxidative stress [4]. Some studies have demonstrated that oxidative stress is linked to a variety of human diseases, such as peritoneal sepsis, acute lung injury/ARDS as well as liver injury [5-9].

Low-dose ROS could regulate signal transduction, gene expression and fundamental cell activities [10]. However, when excessive amount of ROS leads to cellular damage that can culminate in cellular death [11].SODs constitute a family of antioxidant enzymes that includes three SOD isoforms with precise compartmentalization. Cu/Zn-dependent isoform $(\mathrm{Cu} / \mathrm{Zn}-\mathrm{SOD}$, SOD1) which is located in cytosol, the Mn-dependent isoform (Mn-SOD, SOD2) which is located in the mitochondrial matrix and ECSOD is located in the extracellular space. Oxidative stress may lead to lipid peroxidation and an increase in MDA accumulation, and may cause cytotoxicity [12].

Ulinastatin, a serine protease inhibitor,scavenge oxygen free radicals and eliminate inflammatory cytokines to ultimately protect organ functions from various harmful factors [13-16]. It has been used in the treatment of shock, sepsis, acute lung injury, and pancreatitis.[17-21]. Many preclinical studies have shown an observably reduction in the systemic inflammatory response and organ dysfunction due to sepsis in animals treated with UTI [22]. Several clinical studies have shown that UTI can reduce mortality and duration of hospitalization in severe sepsis [23]. The increase in oxidative stress is a central feature observed in the sepsis. JNK/c-Jun signaling has been demonstrated to play an essential role in it [24].

A variety of stress stimulate JNK activation and in turn phosphorylate several transcription factors, such as JunB, ATF-2 and c-Jun, required for cell survival, transformation, proliferation, and death [25,26]. C-Jun is one component of the activator protein 1(AP-1) and JNK can phosphorylate c-Jun within its transcriptional activation domain. SP600125, the inhibitor of JNK, 
can repress c-Jun phosphorylation. LPS induces the expression of ROS production that activates JNK and its substrates c-Jun

[27]. ROS has been characterized as an important secondary messenger and modulator for the JNK/c-Jun pathways.

However, the potential molecular mechanism underlying UTI attenuates oxidative stress was not fully studied. Based on these previous findings, we hypothesize that UTI attenuates LPS-induced oxidative stress in HUVECs via JNK/c-Jun signaling pathway by inhibiting phosphorylation of JNK/c-Jun.

\section{Materials and Methods}

\section{Ethical approval}

This study has been approved by the ethical committee of Shandong Provincial Hospital of Shandong University, and informed consent was obtained from all participants.

\section{Cell Culture}

HUVECs were isolated from human umbilical cords with collagenase as described previously [28], The cord was severed from the placenta soon after birth, cannulated and perfused with $0.1 \%$ collagenase type II in the umbilical vein. After dissociation, the cells were collected and cultured on gelatin-coated culture dishes in endothelial cell culture medium with low serum growth supplement, $100 \mathrm{IU} / \mathrm{ml}$ penicillin, and $0.1 \mathrm{mg} / \mathrm{ml}$ streptomycin, in a humidified atmosphere containing $5 \% \mathrm{CO}_{2}$ and $95 \%$ air at $37^{\circ} \mathrm{C}$. Subcultures were performed with $0.25 \%$ trypsin and $0.01 \%$ EDTA when $80 \%$ confluent. Studies with HUVECs were conducted with cells from passage two to five.The identity of umbilical vein endothelial cells was confirmed by their cobblestone morphology and strong positive immunoreactivity to von Willebrand factor. The umbilical cords were collected from Department of Obstetrics, Shandong Provincial Hospital of Shandong University.

\section{MTT Cell Viability Assay}

Viability and growth patterns of HUVECs were determined by 3-[4, 5-dimethylthiazol-2-yl]-2, 5-Diphenyltetrazolium bromide 
(MTT) Assay (Jiancheng, Nanjing, China) . Briefly, HUVECs were seeded on 96-well plates (1 x $10^{5}$ cells/well), 100 $\mu$ l of MTT solution $(0.5 \mathrm{mg} / \mathrm{ml}$ in the medium) was added to HUVECs treated with various concentrations of LPS(Sigma-Aldrich,USA) for $24 \mathrm{~h}$ each well, and the plates were incubated at $37^{\circ} \mathrm{C}$ for additional $4 \mathrm{~h}$. Afterwards, the medium containing MTT was removed and the crystals were dissolved in $150 \mu \mathrm{l}$ of $100 \%$ dimethyl sulfoxide (DMSO). The cell viability wasdetermined by measuring the optical density (OD) at $570 \mathrm{~nm}$ using a quantified microplate reader (Bio-Rad, Hercules, CA, USA). All experiments were performed in three wells and repeated for three times.

\section{Western blot analysis}

HUVECs were treated with or without LPS at the indicated concentration and time after pre-incubation with the indicated concentrations of UTI for $2 \mathrm{~h}$. Cells were harvested and lysed by scraping in ice-cold RIPA buffer (Santa Cruz, USA). Protein concentrations were determined by BCA assay (Solarbio, China) and total protein for each sample 50ug were resolved on 4-15\% Tris-HCl SDS-polyacrylamide gels and blotted onto Immobilon PVDF for immunoblotting. The blots were incubated with 5\% non-fat dry milk in TBST for $1 \mathrm{~h}$ at room temperature and then stained with anti-human Cu/Zn-SOD, Mn-SOD (1:5000) (Sigma Aldrich, USA), anti-phospho-JNK (1:1000), anti-JNK (1:1000), anti-c-Jun (1:1000), anti-phospho-c-Jun (1:1000)(Cell Signaling Technology, Beverly, MA, USA) ,and anti mouse GAPDH antibody (1:5000 dilution) overnight at $4^{\circ} \mathrm{C}$. After incubation with primary antibodies, membranes were washed, incubated with horseradish secondary antibody (1:5000) for $1 \mathrm{~h}$, and washed again. Protein band densities were quantified using an enhanced chemiluminescence detection system (syngene, USA). The protein levels were analyzed using the Image $\mathbf{J}$ software.

\section{Measurement of reactive oxygen species}

The effect of UTI on ROS production in HUVECs was determined by a fluorometric assay using Abcam's Cellular Reactive Oxygen Species Detection Assay Kit (Abcam, Cambridge, UK)..After pre-incubation with the indicated concentrations of UTI for $2 \mathrm{~h}$, and $10 \mu \mathrm{M} \mathrm{SP} 60025$ or the ROS scavenger (NAC 10mM) (Sigma-Aldrich, USA) for 1h. HUVECs were incubated with the ROS Deep Red assay solution for $1 \mathrm{~h}$ followed by incubation with LPS for $2 \mathrm{~h}$. Cell pellets were washed with PBS and then 
cells lysed in deionized water. Cells were handled in the dark throughout the experiment from cell culture to the fluorescence microscopy measurements. Within the cell, the ROS Deep Red Dye is cell-permeable which generated Deep Red fluorescence when it reacted with ROS. The fluorescence signal of the ROS Deep Red Dye was measured by fluorescence microscopy (Nikon, Japan).

\section{Assay for intracellular malondialdehyde (MDA)}

MDA is the end product of polyunsaturated fatty acid peroxide degeneration. It can be detected with colorimetry. The cells were kept in lysate on ice for 10 minutes, taken out with a spatula, mixed, and centrifuged at $4^{\circ} \mathrm{C} 14000 \mathrm{x} \mathrm{g}$ for $15 \mathrm{minutes}$. The supernatant was then treated with BCA kit to detect its protein concentration. Subsequently, $100 \mu$ protein sample was mixed with $200 \mu 1$ operative solution, bathed in boiling water for 15 minutes, cooled, and then centrifuged with $1000 \mathrm{x} g$ for $10 \mathrm{minutes}$ under room temperature. $200 \mu \mathrm{l}$ supernatant was used to measure its absorbance at $532 \mathrm{~nm}$ using a 96 -pole plate and a microplate reader. The concentration of $\mathrm{MDA}(\mu \mathrm{mol} / \mathrm{mg})$ was calculated according to the standard curve.

\section{Statistical analyses}

Data were expressed as mean \pm SD $(n \geq 3)$. Comparison of the means was performed by one-way analysis of variance (ANOVA), and SPSS version 11.0 software was employed. The results were analyzed statistically using Prism5 (GraphPad Software). $\mathrm{p}<0.05$ was considered statistically significant.

\section{Results}

LPS induces oxidative stress and JNK and c-Jun phosphorylation dose-dependently in

\section{HUVECs}

LPS rapidly led to decrease of SOD1 expression and increase of SOD2 expression compared with control in a dose-dependent manner (Fig.1A). LPS resulted in a significant increase of MDA levels (Fig.1B).JNK and c-Jun phosphorylation was significantly enhanced by LPS in a dose-dependent manner, with a maximal peak at $10 \mu \mathrm{g} / \mathrm{ml}(* * \mathrm{p}<0.01)$. However, total proteins of both JNK and c-Jun remained unaltered after treatment with LPS (Fig.1C). Cell viability was evaluated by a MTT assay, and it 
was found that LPS decreased cell viability in a dose-dependent manner and the percentage of living cells decreased along with the increase of LPS concentration (Fig.1D). Cell viability was not changed significantly when HUVECs were exposed to 0.1 or $1 \mu \mathrm{g} / \mathrm{ml}$ LPS. However, HUVECs were almost dead when treated with $20 \mu \mathrm{g} / \mathrm{ml}$ LPS for $24 \mathrm{~h}$. Therefore, we used $10 \mu \mathrm{g} / \mathrm{ml}$ LPS in the following experiments.

LPS induces oxidative stress and JNK and c-Jun phosphorylation time-dependently in

\section{HUVECs}

LPS rapidly led to decrease of SOD1 expression and to increase of SOD2 expression compared to control group, with the most significant variation observed at $24 \mathrm{~h}$ and $48 \mathrm{~h}\left({ }^{*} \mathrm{p}<0.05\right)(* * \mathrm{p}<0.01)$ (Fig $\left.2 \mathrm{~A}\right)$.Compared with control cells, the levels of MDA have a significant increase at $24 \mathrm{~h}$ and $48 \mathrm{~h}\left({ }^{* *} \mathrm{p}<0.01\right)$ (Fig 2B). JNK and c-Jun were phosphorylated in a time-dependent manner, with the peak at $24 \mathrm{~h}(* * \mathrm{p}<0.01)$ (Fig 2C).Cell viability was evaluated by a MTT assay, and LPS decreased cell viability in a time-dependent manner (Fig 2D). However, HUVECs were almost dead when treated with $10 \mu \mathrm{g} / \mathrm{ml}$ LPS for 48h. Therefore, the treatment time of $24 \mathrm{~h}$ was used in the following experiments.

\section{Effects of Ulinastatin on LPS-induced oxidative stress}

UTI pretreatment significantly reversed the reduction in SOD1 protein expression caused by LPS but showed no effect on SOD2 protein expression (Fig 3A). Pretreatment with UTI can inhibit LPS stimulated ROS level in HUVECs (Fig 3B).To determine the role of JNK/c-Jun signal pathway in the protection of UTI against LPS-provoked oxidative stress . Pretreatment with UTI before exposure to LPS resulted in suppression of JNK/c-Jun phosphorylation. However, total JNK protein and total c-Jun protein remained unaltered, indicating that UTI $(100 \mathrm{U} / \mathrm{ml})$ was the most effective concentration $(* * \mathrm{p}<0.01)$. In addition, with the increasing concentration of UTI, the inhibition on the phosphorylation of JNK/c-Jun decreased (Fig 3C). Pretreatment with UTI can prevent the increase of LPS-induced MDA levels (Fig 3D). These results suggested that UTI can suppress JNK/c-Jun phosphorylation in HUVECs. To exclude an ability of UTI to induce cell death, we investigated the effect of UTI on cell viability using MTT assay. Loss of viability was not observed following UTI exposure at various doses (50-400 U/ml) after 24 h (data 
were not shown).

\section{Effects of JNK/c-Jun signal pathway on LPS-Induced oxidative stress in HUVECs}

LPS significantly reduced SOD1 protein expression and enhanced SOD2 protein expression. Pretreatment with UTI or SP600125 significantly reversed the reduction of SOD1expression caused by LPS but showed no effect on SOD2 protein expression(Fig 4A) .Pretreatment with UTI or JNK SP600125 before exposure to LPS suppressed JNK/c-Jun phosphorylation. However, total JNK protein and total c-Jun protein remained unaltered after treatment with UTI, SP600125 and LPS (Fig 4B). Stimulated with LPS, the intracellular ROS level in HUVECs increased rapidly, causing oxidative stress. We found that treatment with antioxidant NAC (10mM) or SP600125 showed a significant inhibitory effect on the LPS-induced ROS overproduction, and caused a reduction of JNK/c-Jun phosphorylation in LPS-stimulated HUVECs (Fig 4C,4D), suggesting that ROS generation, one of the earliest signals after LPS exposure, was largely dependent on the JNK phosphorylation. Pretreatment with UTI or SP600125 notably prevented the increase of LPS-induced MDA levels (Fig 4E).

\section{Discussion}

In the present study, we found that LPS induced profound elevation of ROS production, increase of MDA levels and decreases

SOD1 . The results indicated that the levels of JNK and c-Jun protein as well as JNK/c Jun protein phosphorylation were

regulated by LPS in HUVECs in a time and dose-dependent manner demonstrated by a Western blot analysis. UTI can

significantly suppress the phosphorylation of JNK induced by LPS in dose-dependent fashion, in accordance with the inhibition

of c-Jun that occurred prior to oxidative stress. SP600125 is an inhibitor of JNK, we found it could markedly suppress

oxidative stress. Taken together, these results provide an evidence that UTI could play an antioxidant effect in HUVECs by

inhibiting phosphorylation of JNK and c-Jun protein.

MAPKs are important kinases in HUVECs redox signaling, JNK is known as stress-activated protein kinase and play key roles in inflammation, cellular stress, and apoptosis. It is regulated predominantly by c-Jun in downstream of cell signaling pathways

[30]. Our results showed that LPS treatment potentiated the phosphorylation of JNK/c-Jun in a dose- and time-dependent manner 
without the alternations of JNK/ c-Jun total protein, which was consistent with other studies that LPS affected MAPK signaling pathways in some other cell types [31].The results from our present study clearly shown that JNK signaling pathway is involved in mediating anti-oxidative stress by UTI. In addition, this inhibition was confirmed by using SP600125. So we can conclude that the mechanism of anti-oxidant effect of UTI is, at least in part, through activation of the JNK signaling pathway.

Low-dose ROS function as signaling molecules to activate various signaling pathways, including the MAPK pathway [32], while phosphorylated MAPKs also could produce more ROS. When we stimulated cells with LPS, the intracellular ROS level in HUVECs increased rapidly and enhance JNK/c-Jun phosphorylation. We found that treatment with the ROS scavenger (NAC) resulted in a reduction in JNK/c-Jun phosphorylation in LPS-stimulated HUVECs. Therefore, JNK is downstream signaling of ROS. Furthermore, LPS-induced ROS was abolished by pretreatment with SP600125, suggesting that ROS generation, one of the earliest signals after LPS exposure, was largely dependent on the JNK phosphorylation. These results clearly suggest that ROS work both upstream and downstream of the JNK activation in LPS-treated HUVECs.

In this study, LPS down-regulated SOD1 protein in time and dose-dependent manner was observed. SOD1 inhibition may result in a decrease in the protective effect of SOD1 against oxidative stress. Our results showed that inhibition of the JNK pathway by pretreatment of SP600125 in HUVECs prevented SOD1 down-regulation induce by LPS, suggesting that JNK/c-Jun acts as promoter or activator of SOD1. Up-regulation of SOD2 expression by LPS is another significant finding in our study, SOD2 is one of the critical antioxidant enzymes in living cells. We documented that LPS caused a time- and dose-dependent manner in up-regulating SOD2 protein expression in HUVECs. SOD is ubiquitously present in different organelles within the cell. SOD1 is present in the cytosol. SOD2, a manganese containing enzyme, is present in the mitochondrial matrix [33]. In this study, we found that UTI and SP600125 pretreatment significantly reversed the reduction in SOD1 expression caused by LPS, but showed no effect on SOD2 protein expression. This suggests that HUVECs may be able to withstand oxidative stress by increasing expression and activity of SOD2, mechanisms responsible for this remain to be investigated. Our findings differ from previous reports in that the increased of SOD2 protein expression is specific to HUVECs [34]. The reason for the different 
response in HUVECs may be related to the lower basal expression of SOD2 in these cells compared to other cell types [35].

LPS may cause lipid peroxidation and an increase in the malondialdehyde (MDA) concentration, and may induce cytotoxicity.

The level of MDA represents the intensity of cell injury [12]. These changes of membrane lipid composition may be induced by free radical initiated lipid peroxidation. In our study, UTI exhibited efficacy for inhibiting MDA levels induced by LPS.

We are aware of the limitations of the experimental model .Although the LPS model is widely used in experiment, limitations in vitro do not allow an exact replication of human variability and comorbidity in human sepsis. In the future, we will validate our findings in vivo.

In summary, we have demonstrated that the anti-oxidant of UTI observed in HUVECs induced by LPS repress through JNK/c-Jun signaling pathway. The data presented here support JNK/c-Jun is a potential therapeutic target for the treatment of sepsis and that inhibiting oxidative stress with UTI administration. Furthermore, elucidating the anti-oxidant mechanism of UTI in sepsis allows for a potential treatment either as single agent or in combination with other known sepsis therapeutics.

\section{Acknowledgements}

This work was supported by Foundation of Tian Pu (Grant no.01201005), Project supported by the National Science Foundation for Distinguished Young Scholars of China (Grant no.81200238) and Project supported by the National Science Foundation of China (Grant no.81372473) 


\section{References}

1. Matsumoto H, Yamakawa K, Ogura H, Koh T, Matsumoto N and Shimazu T (2015) Enhanced Expression of Cell-Specific Surface Antigens on Endothelial Microparticles in Sepsis-Induced Disseminated Intravascular Coagulation. Shock 43:443-9. doi: 10.1097/shk.0000000000000331

2. Dixit D, Ghildiyal R, Anto NP and Sen E (2014) Chaetocin-induced ROS-mediated apoptosis involves ATM-YAP1 axis and JNK-dependent inhibition of glucose metabolism. Cell Death Dis 5:e1212-. doi: 10.1038/cddis.2014.179

3. Tang H, Ge H, Chen ZB, Luo XM, Su FJ, Liang YB, Li ZY, Wu JG, Yang Q, (2015) Micrometam C Protects against Oxidative Stress in Inflammation Models in Zebrafish and RAW264.7 Macrophages. Mar Drugs 13:5593-605. doi: $10.3390 / \mathrm{md} 13095593$

4. Zhang M, Wang A, He W, He P, Xu B, Xia T, Chen X and Yang K (2007) Effects of fluoride on the expression of NCAM, oxidative stress, and apoptosis in primary cultured hippocampal neurons. Toxicology 236:208-16. doi: 10.1016/j.tox.2007.04.007

5. Wang J, He C, Wu WY, Chen F, Wu YY, Li WZ, Chen HQ and Yin YY (2015) Biochanin A protects dopaminergic neurons against lipopolysaccharide-induced damage and oxidative stress in a rat model of Parkinson's disease. Pharmacol Biochem Behav 138:96-103. doi: 10.1016/j.pbb.2015.09.013

6. Zhao J, Wei J, Weathington N, Jacko AM, Huang H, Tsung A and Zhao Y (2015) Lysophosphatidic acid receptor 1 antagonist ki16425 blunts abdominal and systemic inflammation in a mouse model of peritoneal sepsis. Transl Res 166:80-8. doi: 10.1016/j.trs1.2015.01.008

7. Jiang W, Luo F, Lu Q, Liu J, Li P, Wang X, Fu Y, Hao K, Yan T and Ding X (2015) The protective effect of Trillin LPS-induced acute lung injury by the regulations of inflammation and oxidative state. Chem Biol Interact. doi: 10.1016/j.cbi.2015.09.010

8. Liao WQ, Qi YL, Wang L, Dong XM, Xu T, Ding CD, Liu R, Liang WC, Lu LT, Li WF, Luo GB and Lu XC (2015) Recq15 protects against lipopolysaccharide/D-galactosamine-induced liver injury in mice. World J Gastroenterol 21:10375-84. 
doi: 10.3748/wjg.v21.i36.10375

9. Wang G, Wen J, Wilbur RR, Wen P, Zhou SF and Xiao X (2013) The effect of somatostatin, ulinastatin and Salvia miltiorrhiza on severe acute pancreatitis treatment. Am J Med Sci 346:371-6. doi: 10.1097/MAJ.0b013e31827aa2bc

10. Lum H and Roebuck KA (2001) Oxidant stress and endothelial cell dysfunction. Am J Physiol Cell Physiol 280:C719-41.

11. Kao CL, Chen LK, Chang YL, Yung MC, Hsu CC, Chen YC,Chen SJ, Ku HH and Hwang SJ (2010) Resveratrol protects human endothelium from $\mathrm{H}(2) \mathrm{O}(2)$-induced oxidative stress and senescence via SirT1 activation. J Atheroscler Thromb $17: 970-9$

12. Aytekin I, Aksit H, Sait A, Kaya F, Aksit D, Gokmen M and Baca AU (2015) Evaluation of oxidative stress via total antioxidant status, sialic acid, malondialdehyde and RT-PCR findings in sheep affected with bluetongue. Vet Rec Open 2. doi: 10.1136/vetreco-2014-000054

13. Bae HB, Jeong CW, Li M, Kim HS and Kwak SH (2012) Effects of urinary trypsin inhibitor on lipopolysaccharide-induced acute lung injury in rabbits. Inflammation 35:176-82. doi: 10.1007/s10753-011-9303-y

14. Wang N, Liu X, Zheng X, Cao H, Wei G, Zhu Y, Fan S, Zhou H and Zheng J (2013) Ulinastatin is a novel candidate drug for sepsis and secondary acute lung injury, evidence from an optimized CLP rat model. Int Immunopharmacol 17:799-807. doi: 10.1016/j.intimp.2013.09.00417.

15. Inoue $\mathrm{K}$ and Takano H (2010) Urinary trypsin inhibitor as a therapeutic option for endotoxin-related inflammatory disorders. Expert Opin Investig Drugs 19:513-20. doi: 10.1517/13543781003649533

16. Ito K, Mizutani A, Kira S, Mori M, Iwasaka H and Noguchi T (2005) Effect of Ulinastatin, a human urinary trypsin inhibitor, on the oleic acid-induced acute lung injury in rats via the inhibition of activated leukocytes. Injury 36:387-94. doi: 10.1016/j.injury.2004.06.018

17. Wang SY, Li ZJ, Wang X, Li WF and Lin ZF (2015) Effect of ulinastatin on HMGB1 expression in rats with acute lung injury induced by sepsis. Genet Mol Res 14:4344-53. doi: 10.4238/2015.April.30.7 
18. Liu B, Huang W, Xiao X, Xu Y, Ma S and Xia Z (2015) Neuroprotective Effect of Ulinastatin on Spinal Cord Ischemia-Reperfusion Injury in Rabbits. Oxid Med Cell Longev 2015:624819. doi: 10.1155/2015/624819

19. Guo H, Chen J and Suo D (2015) [Clinical efficacy and safety of ulinastatin plus octreotide for patients with severe acute pancreatitis]. Zhonghua Yi Xue Za Zhi 95:1471-4.

20. Zhou GB, Xu QL, Liu YA, Wang ZL, Su L and Guo XH (2015) [Protective effects of ulinastatin against acute lung injury induced by heatstroke in mice]. Nan Fang Yi Ke Da Xue Xue Bao 35:1277-82.

21. Koga Y, Fujita M, Tsuruta R, Koda Y, Nakahara T, Yagi T,Yuasa M and Maekawa T (2010) Urinary trypsin inhibitor suppresses excessive superoxide anion radical generation in blood, oxidative stress, early inflammation, and endothelial injury in forebrain ischemia/reperfusion rats. Neurol Res 32:925-32. doi: 10.1179/016164110x12645013515133

22. Huang N, Wang F, Wang Y, Hou J, Li J and Deng X (2013) Ulinastatin improves survival of septic mice by suppressing inflammatory response and lymphocyte apoptosis. J Surg Res 182:296-302. doi: 10.1016/j.jss.2012.10.043

23. Karnad DR, Bhadade R, Verma PK, Moulick ND, Daga MK, Chafekar ND and Iyer S (2014) Intravenous administration of ulinastatin (human urinary trypsin inhibitor) in severe sepsis: a multicenter randomized controlled study. Intensive Care Med 40:830-8. doi: 10.1007/s00134-014-3278-8

24. Zhao E, Amir M, Lin Y and Czaja MJ (2014) Stathmin Mediates Hepatocyte Resistance to Death from Oxidative Stress by down Regulating JNK. PLoS One 9. doi: 10.1371/journal.pone.0109750

25. Dong C, Yang DD, Wysk M, Whitmarsh AJ, Davis RJ and Flavell RA (1998) Defective T cell differentiation in the absence of Jnk1. Science 282:2092-5.

26. Ma J, Zhang L, Han W, Shen T, Ma C, Nie X, Ran Y and Zhu D (2012) Activation of JNK/c-Jun is required for the proliferation, survival, and angiogenesis induced by EET in pulmonary artery endothelial cells[S]. J Lipid Res 53:1093-105. doi: 10.1194/jlr.M024398

27. Rockwell P, Martinez J, Papa L and Gomes E (2004) Redox regulates COX-2 upregulation and cell death in the neuronal 
response to cadmium. Cell Signal 16:343-53.

28. Lee CH, Lee SD, Ou HC and Cheng YJ (2014) Eicosapentaenoic Acid Protects against Palmitic Acid-Induced Endothelial Dysfunction via Activation of the AMPK/eNOS Pathway. Int J Mol Sci 15:10334-49. doi: 10.3390/ijms150610334

29. Wang H, Sun X, Gao F, Zhong B, Zhang Y and Sun Z (2012) Effect of ulinastatin on growth inhibition, apoptosis of breast carcinoma cells is related to a decrease in signal conduction of JNk-2 and NF- $\mathrm{BB}$. J Exp Clin Cancer Res 31:2. doi: $10.1186 / 1756-9966-31-2$

30. Goldsmith CS and Bell-Pedersen D (2013) Diverse roles for MAPK signaling in circadian clocks. Adv Genet 84:1-39. doi: 10.1016/b978-0-12-407703-4.00001-3

31. Chang A, Chen Y, Shen W, Gao R, Zhou W, Yang S, Liu Y, Luo Y, Chuang TH, Sun P, (2015) Ifit1 Protects Against Lipopolysaccharide and D-galactosamine-Induced Fatal Hepatitis by Inhibiting Activation of the JNK Pathway. J Infect Dis 212:1509-20. doi: 10.1093/infdis/jiv221

32. McCubrey JA, Lahair MM and Franklin RA (2006) Reactive oxygen species-induced activation of the MAP kinase signaling pathways. Antioxid Redox Signal 8:1775-89. doi: 10.1089/ars.2006.8.1775

33. Van Raamsdonk JM and Hekimi S (2009) Deletion of the Mitochondrial Superoxide Dismutase sod-2 Extends Lifespan in Caenorhabditis elegans. PLoS Genet 5. doi: 10.1371/journal.pgen.1000361

34. Cai H, Gehrig P, Scott TM, Zimmermann R, Schlapbach R and Zisch AH (2006) MnSOD marks cord blood late outgrowth endothelial cells and accompanies robust resistance to oxidative stress. Biochem Biophys Res Commun 350:364-9. doi: 10.1016/j.bbrc.2006.09.046

35. He T, Peterson TE, Holmuhamedov EL, Terzic A, Caplice NM, Oberley LW and Katusic ZS (2004) Human endothelial progenitor cells tolerate oxidative stress due to intrinsically high expression of manganese superoxide dismutase. Arterioscler Thromb Vasc Biol 24:2021-7. doi: 10.1161/01.ATV.0000142810.27849.8f 


\section{Legends:}

Fig1. (A) Representative Western blot of Cu/Zn-SOD (SOD1) and Mn-SOD (SOD2) protein levels .(B) The content of intracellular MDA production was determined by measuring MDA-TBA compound after incubated with LPS. (C) LPS induced JNK and c-Jun phosphorylation in HUVECs,protein levels were analyzed by Western blot. (D)Changes of cell viability, which was measured by MTT assay of HUVECs exposed to LPS for $24 \mathrm{~h}$. Data were expressed as the mean $\pm \mathrm{SD}$. * indicated $\mathrm{p}<0.05$, and $* *$ indicated $\mathrm{p}<0.01$ compared with control group.

Fig2. (A) Protein expression of Cu/Zn-SOD (SOD1) and Mn-SOD (SOD2) was examined by Western blot. (B) The content of intracellular MDA production was determined by measuring MDA-TBA compound .(C) HUVECs were stimulated with LPS $(10 \mu \mathrm{g} / \mathrm{ml})$, protein expression of phosphorylated-JNK (p-JNK), phosphorylated-c-Jun (p-c-Jun), total JNK and total c-Jun was examined by Western blot. (D) HUVECs viability was detected by MTT assay. Data were expressed as mean \pm SD. * indicated $p<0.05$, and $* *$ indicated $p<0.01$ compared with untreated cells.

Fig3.(A) Protein expression of was examined by Western blot. (B) Cells were incubation with the ROS Deep Red assay solution, and fluorescence images showed the ROS level in cells (400x). (C) Protein expression of phosphorylated-JNK, phosphorylated-c-Jun, total JNK and total c-Jun was examined by Western blot. (D) The content of intracellular MDA production was determined by measuring MDA-TBA compound. HUVECs viability was detected by MTT assay. Data were expressed as mean \pm SD. * indicated $\mathrm{p}<0.05$, and $* *$ indicated $\mathrm{p}<0.01$ compared with control cells. \# indicated $p<0.05$, and \#\# indicated a $p<0.01$ compared with LPS-stimulated HUVECs.

Fig4.(A) Protein expression of Cu/Zn-SOD (SOD1), and Mn-SOD (SOD2) was examined by Western blot. (B) Protein expression of phosphorylated-JNK phosphorylated-c-Jun, total JNK and total c-Jun was examined by Western blot. (C, D) With the ROS Deep Red assay solution, and fluorescence images showed the ROS level in control cells and HUVECs (400x). Protein expression of phosphorylated-JNK and total JNK was examined by Western blot. (E)The content of intracellular MDA production was determined by measuring MDA-TBA compound. Data were expressed as mean $\pm \mathrm{SD}$. * indicated $p<0.05$, and ** 
indicated $p<0.01$ compared with control cells. \# indicated $p<0.05$, and \#\# indicated $p<0.01$ compared with LPS-stimulated HUVECs. 

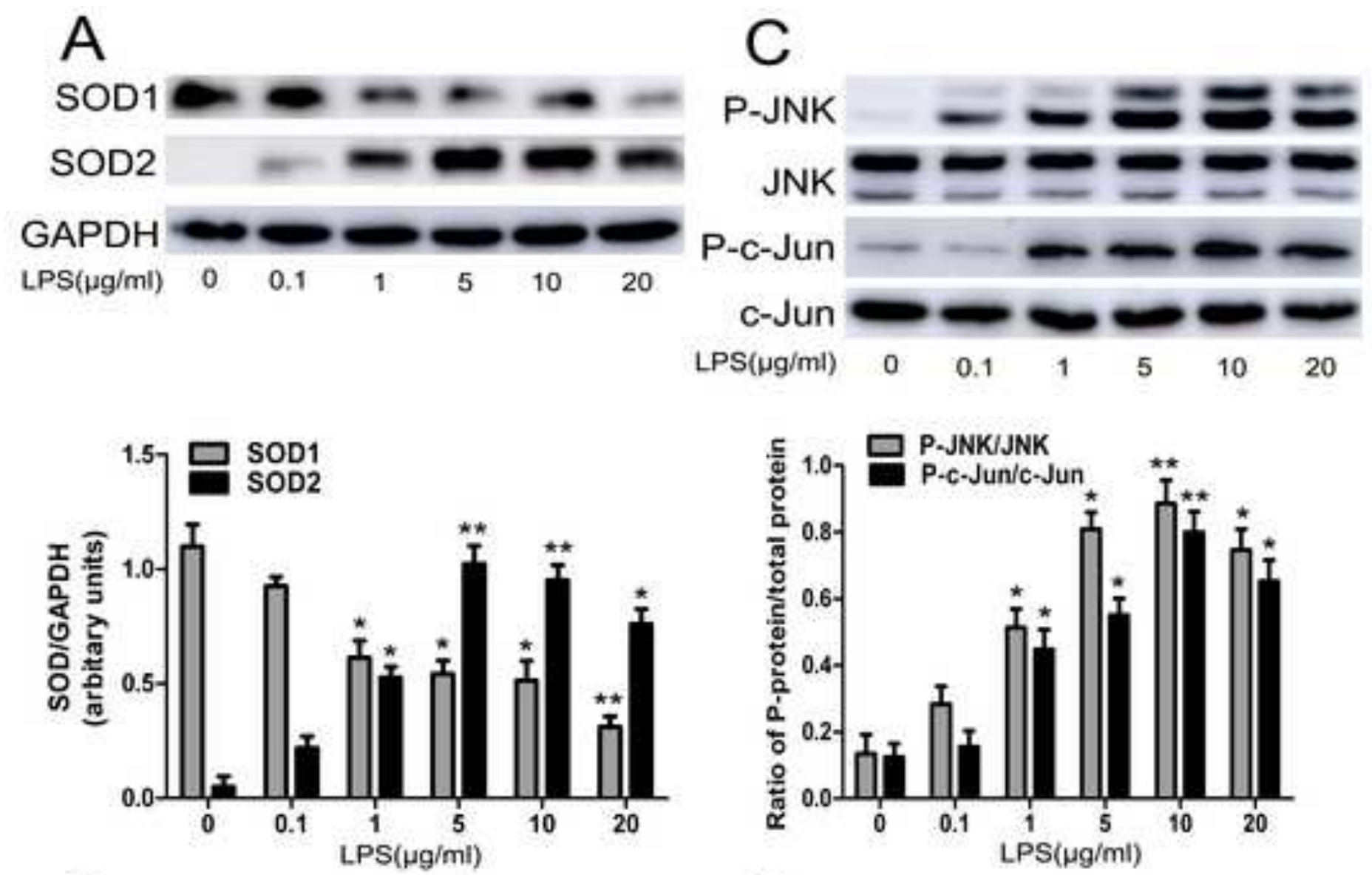

B

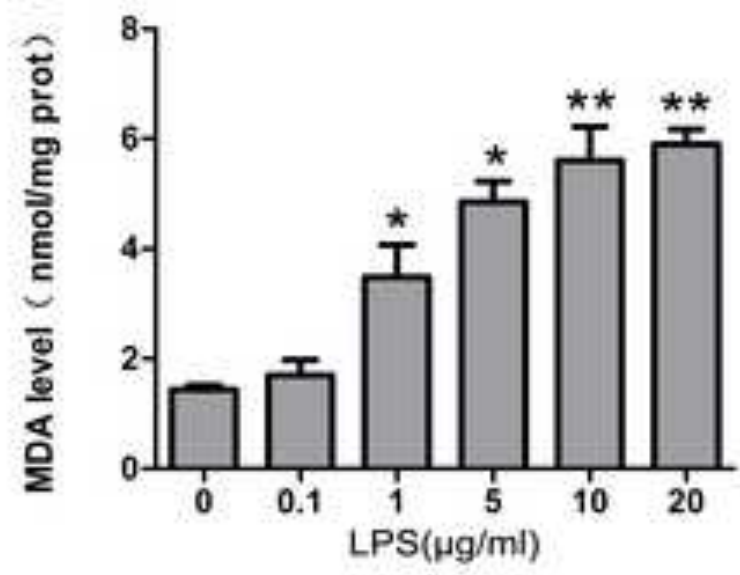

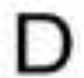

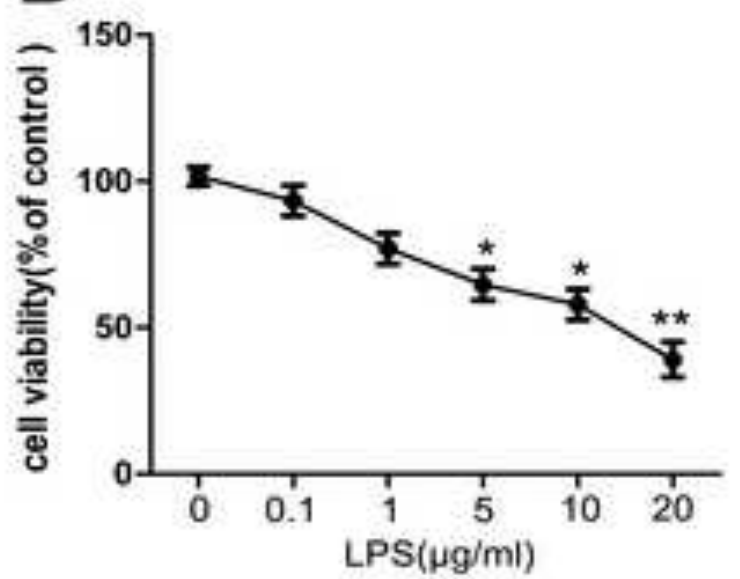


A
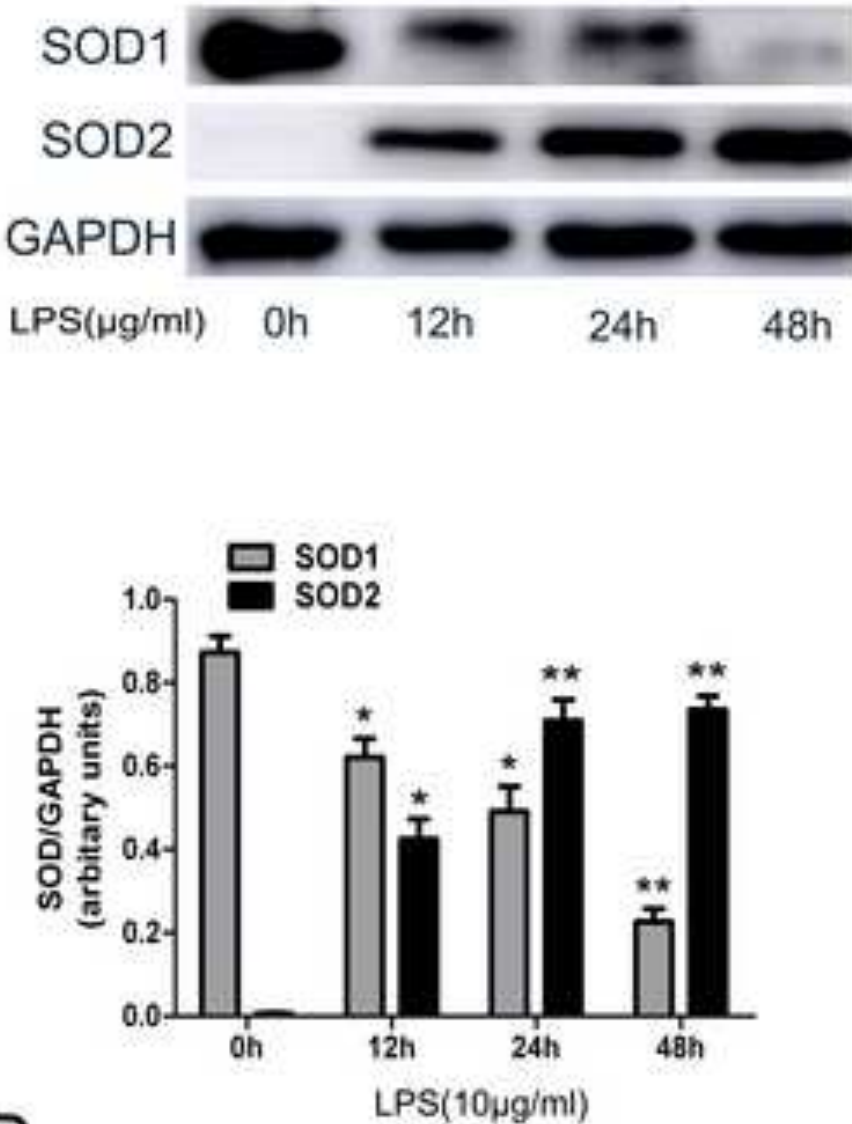

B

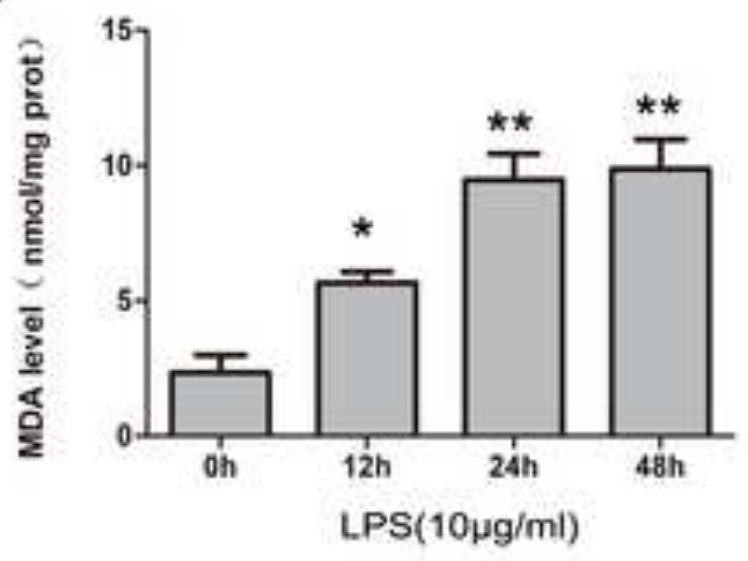

C
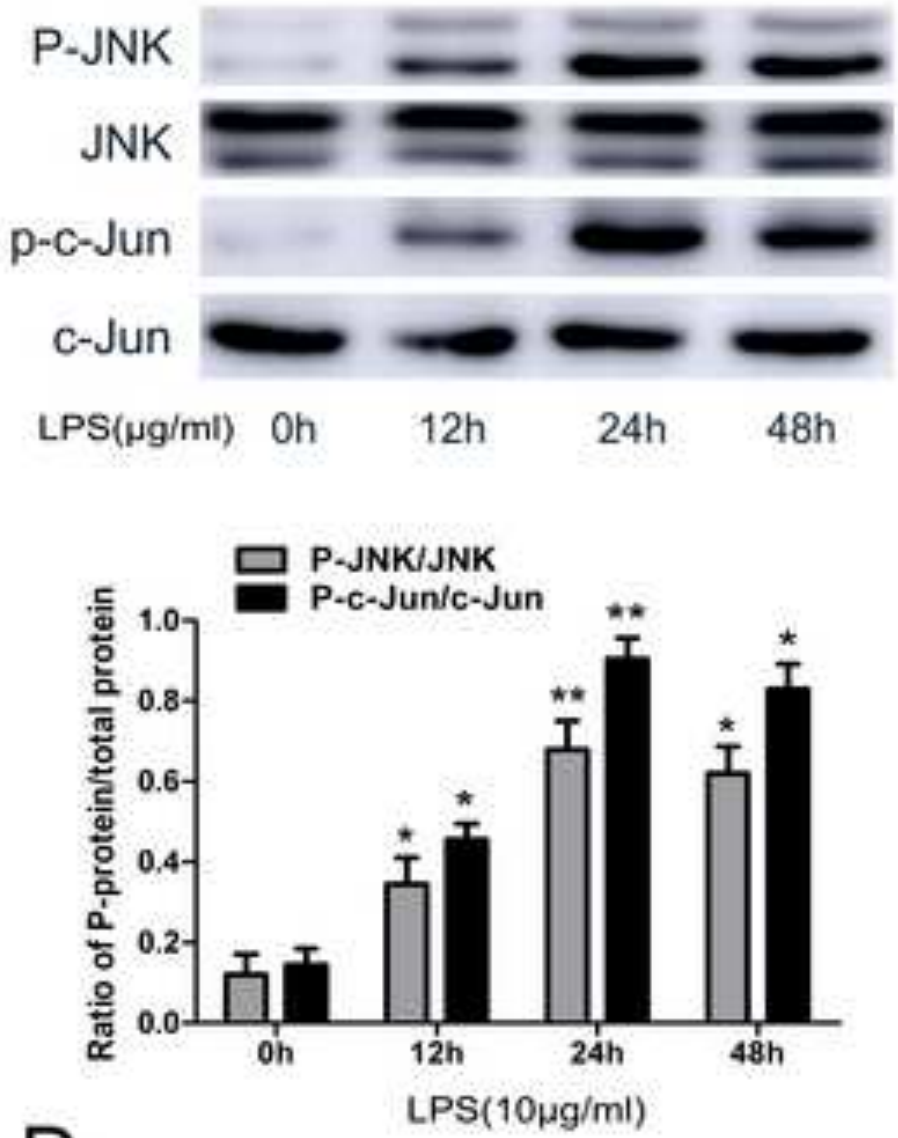

D

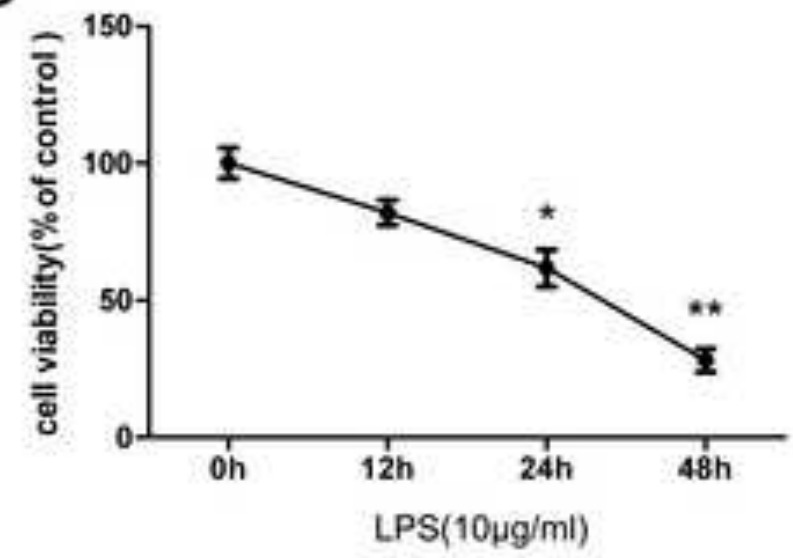




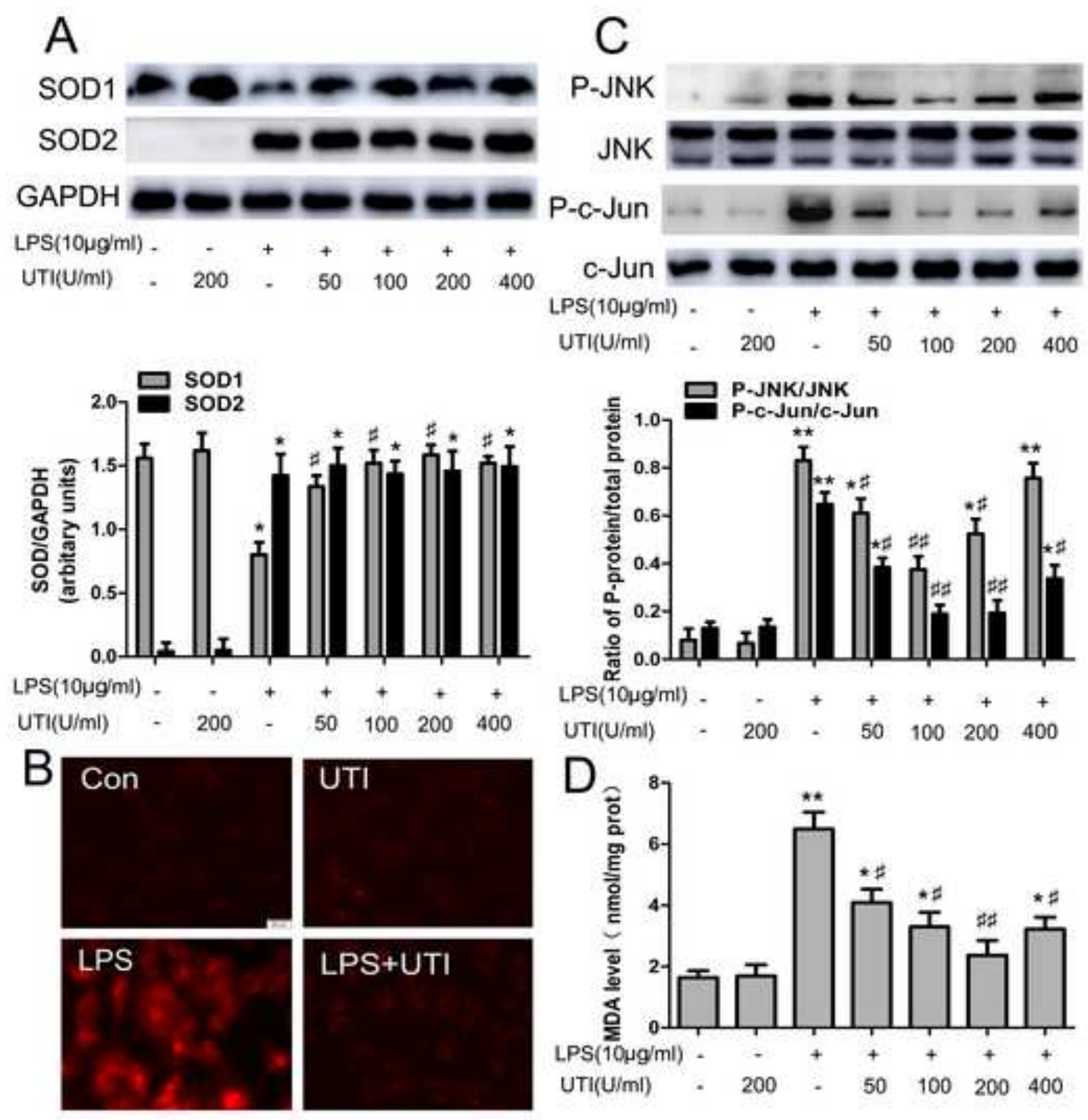




\section{A}

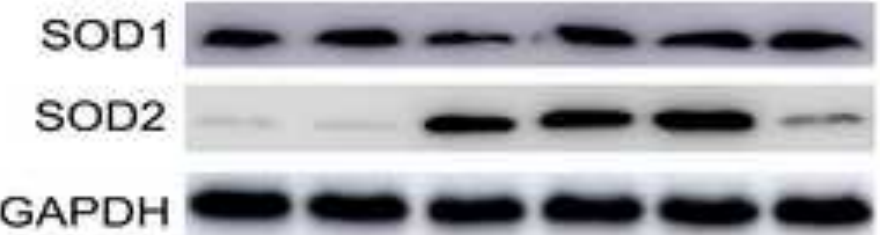

LPS(10ug/ml)

UTI(100U/ml) SP600125

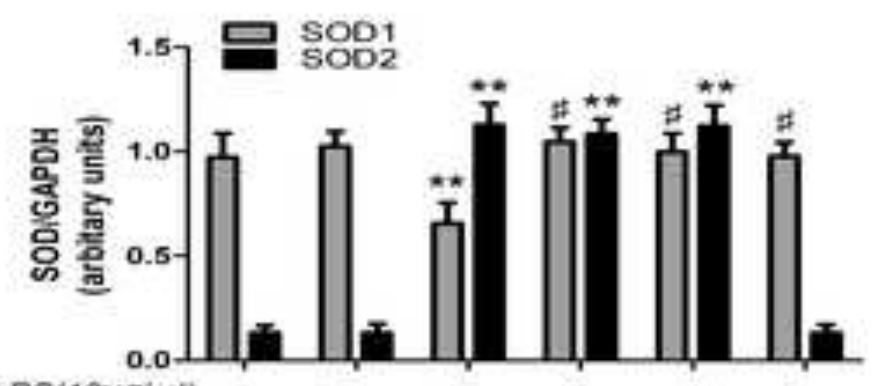

L.PS(10ug/ml)

UTI(100U/mi)

SP600125

C

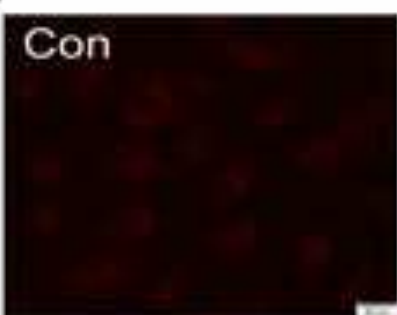

\section{LPS}
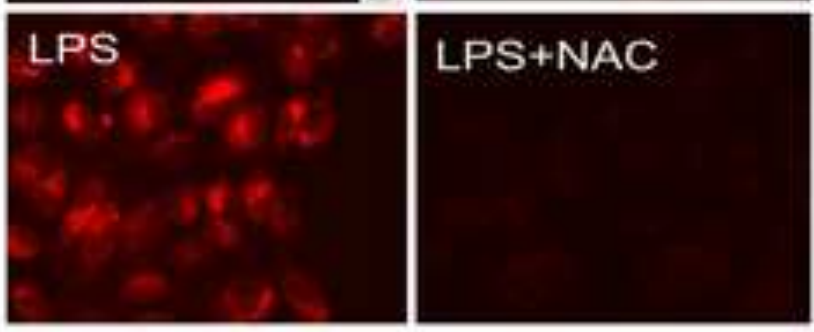

B

P-JNK JNK

P-C-Jun C-Jun

LPS $(10 \mu g / m !)$

UTI(100U/mi)

SP600125

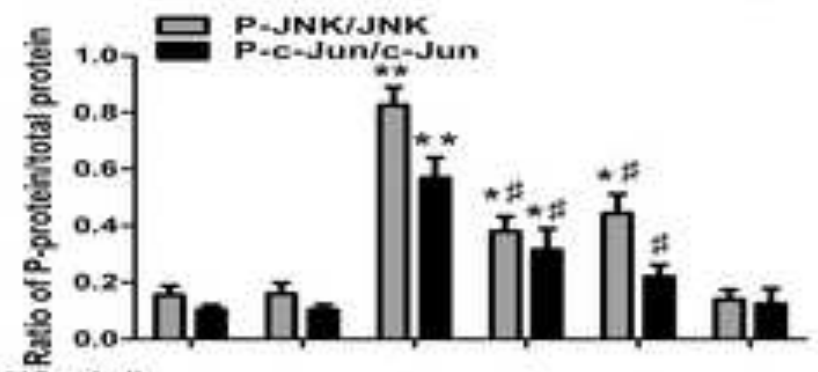

\section{LPS(10 $\mu 9 / m \mid)$}

UTI(100U/mi)

$\mathrm{D}_{\mathrm{P}-\mathrm{JNK}}^{\mathrm{SP600125}}$

$\mathrm{JNK}=-=$

GAPDH

LPS(10pg/m!)

NAC (mM)

E

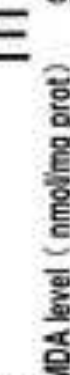

SP600125

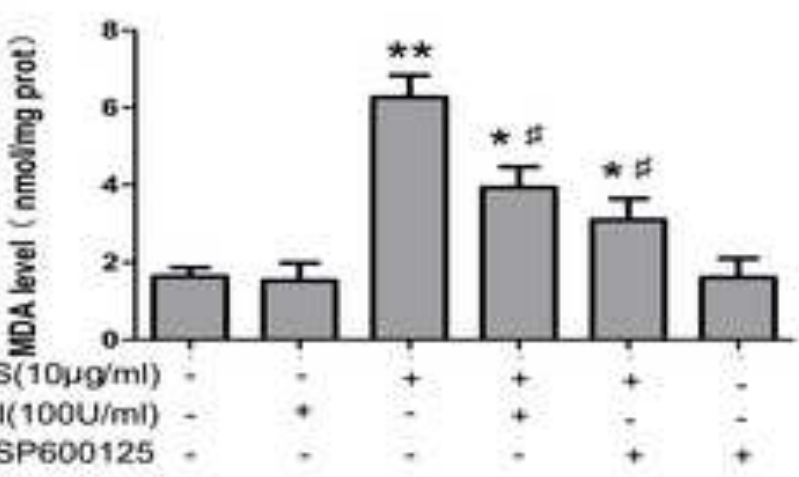

